\title{
Relación entre el tipo de apego y los esquemas maladaptativos tempranos del área de la autonomía en una muestra de niños de 2 a 6 años de edad
}

\author{
Link between attachment type and the early maladaptive schemes of \\ autonomy area in one sample of children from 2 to 6 years of age
}

Relação entre o tipo de acessório e esquemas cedo mal-adaptativos na
área de autonomia em uma amostra de crianças de 2 a 6 anos de idade

Antonio José Villa Londoño ${ }^{2}$

José Luis Álvarez Posada ${ }^{3}$

Recibido: 23.10.2016 - Arbitrado: 20.11.2016 - Aprobado: 10.02.2017

\section{Resumen}

El artículo presenta resultados de una investigación orientada a identificar los tipos de apego y los Esquemas Maladaptativos Tempranos del área de la Autonomía relacionados, en una muestra de niños de 2 a 6 años de edad de la ciudad de Medellín, y determinar las correlaciones estadísticas y psicológicas que sean significativas entre las dos variables. El estudio realizado fue de tipo cuantitativo, nivel correlacional, enfoque empírico analítico. La muestra de participantes fue por conveniencia, y se emplearon como instrumentos y técnicas de recolección de información el cuestionario de Esquemas Maladaptativos Tempranos para niños, y el cuestionario para la identificación del tipo de apego, ambos instrumentos diseñados para la investigación. El estudio brinda datos importantes sobre la relación entre los tipos de apego y los EMT del área de la autonomía, información de utilidad para los psicólogos clínicos. La investigación está enmarcada dentro de la Resolución No 008430 de 1993, por la cual se establecen las normas científicas, técnicas y administrativas para la Investigación en salud.

Palabras clave: Esquemas maladaptativos tempranos, tipo de apego, teoría del apego, autonomía.

\footnotetext{
${ }^{1}$ Investigación realizada en la Universidad de San Buenaventura, Medellín, en 2015

${ }^{2}$ Psicólogo, Universidad de San Buenaventura, Medellín; Especialista, Universidad de San Buenaventura, Medellín; Magister, Universidad de San Buenaventura, Medellín; Doctor en Neurociencias de la Universidad de Maimónides, Argentina; Docente tiempo completo Universidad de San Buenaventura, Medellín, Colombia, anjovi59@gmail.com

${ }^{3}$ Psicólogo, Universidad de San Buenaventura, Medellín; Doctor en Neurociencias de la Universidad de Maimónides, Argentina; Docente tiempo completo Universidad de San Buenaventura, Medellín, Colombia, jlap.cat@gmail.com
}

Citación del artículo: Villa Londoño, A., Álvarez Posada, J. (2017). Relación entre el tipo de apego y los esquemas maladaptativos tempranos del área de la autonomía en una muestra de niños de 2 a 6 años de edad. Revista Katharsis, N 23, enero-julio 2017, pp. 18-33, Disponible en http://revistas.iue.edu.co/index.php/katharsis 


\begin{abstract}
The article presents result of a research aimed at identifying the types of attachment and the Early Maladaptive Schemes of the area of Autonomy related in children from 2 to 6 years of age in the city of Medellín and determine the statistical and psychological correlation that are Between the two variables. The study was of quantitative type, of Correlational level and empirical analytical approach. The sample of participants was for convenience, and the questionnaire for Early Maladaptive Schemes for children and the questionnaire for the identification of the type of attachment, both instruments designed for the investigation, were used as instruments and techniques for collecting information. The study provides important data on the relationship between attachment types and EMSs in the area of autonomy, useful information for clinical psychologists. The research is framed within Resolution No. 008430 of 1993, which establishes the scientific, technical and administrative standards for Health Research.
\end{abstract}

Keywords: Early maladaptive schemes, attachment type, attachment theory, autonomy.

\title{
Resumo
}

$\mathrm{O}$ artigo apresenta os resultados de uma pesquisa que objetivou identificar os tipos de anexos e a área de autonomia precoce adaptativos de esquemas relacionados, numa amostra de crianças de 2 a 6 anos de idade, a cidade de Medellín e determinar as correlações estatísticas e psicológicas que são significativas entre as duas variáveis. O estudo foi a abordagem analítica quantitativa, busca, empírica. A amostra dos participantes foi por conveniência, e eram usados como ferramentas e técnicas de recolha esquemas mal-adaptativos precoce de questionário para as crianças e o questionário para a identificação do tipo de fixação, ambos os instrumentos concebidos para pesquisa de informação. $\mathrm{O}$ estudo fornece fatos importantes sobre a relação entre os tipos de fixação e o EMT da área da autonomia, informação útil para psicólogos clínicos. A pesquisa está enquadrada dentro da resolução n. ${ }^{\circ} 008430$, de 1993, por que estabelecer as regras científicas, técnicas e administrativas para a pesquisa em saúde.

Palavras-Chave: Regimes início mal-adaptativos, acessório, acessório, teoria de autonomia.

\section{Introducción.}

La terapia de las trampas vitales se basa en el desarrollo de un enfoque denominado terapia cognitiva que fue desarrollado por el doctor Aarón Beck en los años sesenta. Jeffrey Young ha desarrollado el enfoque de las trampas vitales (Esquemas Maladaptativos Tempranos) en el que se combinan técnicas cognitivas y conductuales y técnicas experienciales (Young, 2012). Representa una nueva e innovadora ampliación del modelo de la terapia cognitiva de Beck (Caro, 1997)

Citación del artículo: Villa Londoño, A., Álvarez Posada, J. (2017). Relación entre el tipo de apego y los esquemas maladaptativos tempranos del área de la autonomía en una muestra de niños de 2 a 6 años de edad. Revista Katharsis, N 23, enero-julio 2017, pp. 18-33, Disponible en http://revistas.iue.edu.co/index.php/katharsis 
Young establece una relación entre el entorno afectivo en el cual crecen los niños y el desarrollo de EMT's.: estar expuesto al maltrato de los padres, miedo a la soledad por parte de los cuidadores, excesivo control de las emociones, pautas de sobreprotección, tener que cuidar al uno de los padres, incidirían negativamente en el desarrollo de la autonomía de los niños favoreciendo la aparición de EMT’s del área de la autonomía (Young, 2012).

En esta investigación se hace una aproximación desde dicha teoría de los EMT para ampliar la comprensión con respecto a la relación entre los tipos de apego propuestos en la teoría de John Bowlby y los EMT's del área de la autonomía.

En el estudio se busca identificar los tipos de apego y los Esquemas Maladaptativos Tempranos del área de la Autonomía, en una muestra de niños de 2 a 6 años de edad de la ciudad de Medellín, y determinar las correlaciones estadísticas y psicológicas que sean significativas entre las dos variables.

Dicha investigación se ha realizado con el fin de profundizar en el conocimiento de dicha relación y alcanzar una comprensión mayor con respecto a cómo se presentan estas variables en poblaciones de esta ciudad. Igualmente, los resultados brindan información de utilidad para la construcción de planes de prevención e intervención en poblaciones similares a las del estudio que permitan a través de escuelas de padres ayudarles a las figuras de apego a mejorar sus pautas de crianza afectivas y generar así un impacto positivo y duradero en el bienestar y sano desarrollo de los niños que estén a su cuidado.

\section{Referente Teórico.}

\section{Esquemas Maladaptativos Tempranos}

Los EMT's son el resultado de experiencias disfuncionales recurrentes, están estrechamente ligados a altos niveles de afecto y sensaciones corporales, constituyen una abstracción fruto de la experiencia previa, son organizaciones de significados personales referentes al sí mismo, los eventos, las personas y las cosas. Se desarrollan a partir de la relación del niño con su cuidador (Young, 1999).

\section{Área de la Autonomía y Esquemas Maladaptativos Tempranos}

Citación del artículo: Villa Londoño, A., Álvarez Posada, J. (2017). Relación entre el tipo de apego y los esquemas maladaptativos tempranos del área de la autonomía en una muestra de niños de 2 a 6 años de edad. Revista Katharsis, N 23, enero-julio 2017, pp. 18-33, Disponible en http://revistas.iue.edu.co/index.php/katharsis 
Área de la Autonomía: esta área hace referencia a la capacidad del sujeto para funcionar independientemente en el mundo, sin el continuo apoyo de los demás. Los individuos autónomos son capaces de expresar sus necesidades, intereses, preferencias, opiniones y sentimientos. Los EMT de esta área son: Dependencia / Incompetencia, Subyugación / Sumisión Perdida de la Individualidad, Vulnerabilidad al Daño y a la Enfermedad y Temor a Perder el Auto-Control / Inhibición Emocional (Young, 1999).

Procesos de Mantenimiento de Esquemas

Se refiere a los procesos mediante los cuales se refuerzan los esquemas maladaptativos tempranos (EMT). Estos procesos incluyen tanto estilos de pensamiento específicos poco viables, como patrones conductuales de auto derrota relacionados con las profecías autor realizadas (se generan comportamientos que al influir sobre el medio ambiente terminan retroalimentando y confirmando el esquema). También incluyen la rigidez y la dificultad para considerar otras reglas internas de funcionamiento u otras formas de ver el mundo, generando una gran resistencia al cambio (Young, 2012).

Los diferentes tipos de mantenimiento esquemático son:

Mantenimiento o Rendición: sobrevaloración o magnificación de la información que los confirma, comportamientos de auto derrota; Evitación: intentos automáticos o voluntarios por bloquear pensamientos o imágenes que puedan activar el esquema, despersonalización, evitación afectiva, evitación de situaciones de la vida real; Sobrecompensación o Contra ataque: el sujeto termina comportándose de manera totalmente contraria a lo que se esperaría teniendo en cuenta su EMT (Young, 2012).

Tipos de Apego

La pauta de apego seguro: El individuo confía en que sus padres o figuras parentales, serán accesibles, sensibles y colaboradores si él se encuentra en una situación adversa o atemorizante; la pauta de apego ansioso resistente: el individuo esta inseguro de si su progenitor será accesible o sensible o lo ayudará cuando lo necesite; la pauta de apego ansioso elusivo: el individuo no confía en que cuando busque cuidados recibirá una respuesta servicial, sino que, por el contrario, espera ser desairado (Bowlby,1988) la pauta de apego extremo: Las figuras de apego creen que el individuo no es capaz de realizar ninguna actividad por sí solo, lo cual tiene como

Citación del artículo: Villa Londoño, A., Álvarez Posada, J. (2017). Relación entre el tipo de apego y los esquemas maladaptativos tempranos del área de la autonomía en una muestra de niños de 2 a 6 años de edad. Revista Katharsis, N 23, enero-julio 2017, pp. 18-33, Disponible en http://revistas.iue.edu.co/index.php/katharsis 
consecuencia que éste desarrolla una idea pobre de su autoeficacia, asumiendo la creencia de que no es capaz de valerse por sí mismo y es incapaz de tomar una posición autónoma frente a la vida (Ainsworth, 1980).

\section{Metodología.}

Tipo de estudio cuantitativo; nivel correlacional y enfoque empírico analítico.

Alcance: Es una investigación estratégico prospectiva, siendo ésta un estudio que está dirigido a la generación de planes de prevención clínica de análisis de escenarios.

Instrumentos y técnicas de recolección de información: Cuestionario de Esquemas Maladaptativos Tempranos para niños de 2 a 6 años de edad y Cuestionario de Tipo de Apego para niños de 2 a 6 años de edad. Ambos cuestionarios fueron diseñados para la investigación y validados bajo juicio de expertos y aplicados directamente a los cuidadores.

Población: Conformada por 70 niños entre 2 y 6 años de edad, pertenecientes al Jardín Infantil Coqui de Medellín.

Muestra: 70 niños (margen de error $=5$; heterogeneidad=50\%; nivel de confianza $=95 \%$ ).

Criterios de Inclusión: Niños con edades de 2 a 6 años pertenecientes al Jardín Infantil Coqui.

Criterios de Exclusión: Niños con historia previa de discapacidad cognitiva y/o que presenten características del espectro autista.

Variables: Tipo de apego (seguro, ansioso resistente, ansioso elusivo y extremo) y Esquemas Maladaptativos Tempranos (Área de Autonomía).

Hipótesis: Hipótesis Nula $\left(\mathrm{H}_{0}\right)$ "la relación entre las dos variables de datos no es estadísticamente significativa"; Hipótesis Alternativa $\left(\mathrm{H}_{1}\right)$, "la relación entre las dos variables de datos si es estadísticamente significativa"

Procedimiento: La información fue obtenida a partir de la aplicación de los cuestionarios directamente al cuidador de cada niño, luego de obtener los datos se procedió a realizar un análisis estadístico a partir del software SPSS 22 determinando el nivel de asociación entre las variables a través del chi cuadrado, y posteriormente se continuó con el análisis de los resultados.

Citación del artículo: Villa Londoño, A., Álvarez Posada, J. (2017). Relación entre el tipo de apego y los esquemas maladaptativos tempranos del área de la autonomía en una muestra de niños de 2 a 6 años de edad. Revista Katharsis, N 23, enero-julio 2017, pp. 18-33, Disponible en http://revistas.iue.edu.co/index.php/katharsis 


\section{Resultados.}

En el análisis de resultados, además de mostrar dichas relaciones, se hace una aproximación explicativa de las mismas, esto para que sea de utilidad en los contextos de la psicología clínica en donde se abordan las variables aquí investigadas.

La siguiente información fue obtenida a partir del análisis detallado de cada uno de los tipos de apego y los esquemas maladaptativos tempranos del área de la autonomía.

A partir de los objetivos propuestos para la investigación se pudieron identificar los tipos de apego y los EMT de cada uno de los niños participantes en la investigación. Teniendo en cuenta que las variables son de tipo cualitativo (presencia o no de EMT's del área de la autonomía, presencia de determinado tipo de apego), para establecer el nivel de asociación, este se calculó con un chi cuadrado (nivel de significancia $=<0.05$ ).

Pertenecen a esta categoría todas aquellas variables sobre las cuales se hace necesario ejercer un control o manipulación con el fin de que éstas no confundan los resultados obtenidos en el grupo con respecto a las variables a observar. Criterio de edad: de 2 a 6; sexo.

Tabla 1. Medidas de tendencia central de las variables demográficas de los 70 participantes de la muestra.

\begin{tabular}{|c|c|c|c|c|c|}
\hline \multicolumn{6}{|c|}{ Sexo } \\
\hline & & Frecuencia & & $\%$ & \\
\hline Masculino & & 35 & & 50 & \\
\hline Femenino & & 35 & & 50 & \\
\hline Total & & 70 & & 100 & \\
\hline \multicolumn{6}{|c|}{ Edad } \\
\hline & Frecuencia & $\%$ & media & & DS \\
\hline 2 & 11 & 15,7 & & & \\
\hline 3 & 18 & 25,7 & & & \\
\hline 4 & 18 & 25,7 & 3,9 & & 1,287 \\
\hline 5 & 13 & 18,6 & & & \\
\hline 6 & 10 & 14,3 & & & \\
\hline Total & 70 & 100 & & & \\
\hline
\end{tabular}

DS = Desviación Estándar

Citación del artículo: Villa Londoño, A., Álvarez Posada, J. (2017). Relación entre el tipo de apego y los esquemas maladaptativos tempranos del área de la autonomía en una muestra de niños de 2 a 6 años de edad. Revista Katharsis, N 23, enero-julio 2017, pp. 18-33, Disponible en http://revistas.iue.edu.co/index.php/katharsis 


\begin{tabular}{|c|c|c|c|c|}
\hline \multicolumn{5}{|c|}{ Estrato Socioeconómico } \\
\hline Estrato & Frecuencia & $\%$ & media & DS \\
\hline 2 & 14 & 20,0 & \multirow{4}{*}{3,04} & \multirow{4}{*}{0,731} \\
\hline 3 & 42 & 60,0 & & \\
\hline 4 & 11 & 15,7 & & \\
\hline 5 & 3 & 4,3 & & \\
\hline Total & 70 & 100,0 & & \\
\hline
\end{tabular}

Fuente: Autores

La caracterización de la muestra identifica un grupo de personas con una edad media de 3.9 años, con una desviación estándar de 1.287 años; estrato socioeconómico con una media de 3.04 y una desviación estándar de 0.731 .

Tabla 2. Tabla de contingencia Apego Seguro \& EMT’s Autonomía

\begin{tabular}{|c|c|c|c|c|c|c|c|}
\hline \multirow{3}{*}{$\begin{array}{l}\text { VARIABLES DE } \\
\text { ESTUDIO }\end{array}$} & \multirow{3}{*}{ Pte. } & & \multicolumn{4}{|c|}{ Apego Seguro } & \multirow[b]{3}{*}{$\mathrm{p}$} \\
\hline & & $\mathrm{f}$ & \multicolumn{2}{|c|}{$\mathrm{Si}$} & \multicolumn{2}{|c|}{ No } & \\
\hline & & & $\mathrm{n}$ & $\%$ & $\mathrm{n}$ & $\%$ & \\
\hline \multirow{2}{*}{$\begin{array}{l}\text { Dependencia / } \\
\text { Incompetencia }\end{array}$} & $\mathrm{Si}$ & $\mathrm{n}$ & 6 & 17,6 & 6 & 16,7 & \multirow{2}{*}{0,913} \\
\hline & No & $\mathrm{n}$ & 28 & 82,4 & 30 & 83,3 & \\
\hline \multirow{2}{*}{$\begin{array}{l}\text { Vulnerabilidad al } \\
\text { Daño y Enfermedad }\end{array}$} & $\mathrm{Si}$ & $\mathrm{n}$ & 0 & 0,0 & 5 & 13,9 & \multirow{2}{*}{$0,024^{*}$} \\
\hline & No & $\mathrm{n}$ & 34 & 100,0 & 31 & 86,1 & \\
\hline \multirow{2}{*}{$\begin{array}{l}\text { Sumisión / Perdida } \\
\text { Individualidad }\end{array}$} & $\mathrm{Si}$ & $\mathrm{n}$ & 0 & 0,0 & 4 & 11,1 & \multirow{2}{*}{$0,045^{*}$} \\
\hline & No & $\mathrm{n}$ & 34 & 100,0 & 0 & 0,0 & \\
\hline \multirow{2}{*}{$\begin{array}{l}\text { Temor a Perder el } \\
\text { Control / Inhibición } \\
\text { Emocional }\end{array}$} & $\mathrm{Si}$ & $\mathrm{n}$ & 0 & 0,0 & 4 & 11,1 & \multirow[b]{2}{*}{0,045} \\
\hline & No & $\mathrm{n}$ & 34 & 100,0 & 32 & 88,9 & \\
\hline
\end{tabular}

Pte. $=$ presencia de la variable; $\mathrm{f}=$ frecuencia absoluta; $\mathrm{p}$ calculada por el chi cuadrado

Fuente: Autores

Teniendo como base los datos arrojados, se observa un alto nivel de asociación entre la variable de Apego Seguro y la variable EMT's de Vulnerabilidad al Daño y a la Enfermedad, Sumisión / Perdida de la Individualidad y Temor a Perder el Control / Inhibición Emocional (Tabla N 2).

Citación del artículo: Villa Londoño, A., Álvarez Posada, J. (2017). Relación entre el tipo de apego y los esquemas maladaptativos tempranos del área de la autonomía en una muestra de niños de 2 a 6 años de edad. Revista Katharsis, N 23, enero-julio 2017, pp. 18-33, Disponible en http://revistas.iue.edu.co/index.php/katharsis 
Tabla 3: Tabla de contingencia Apego Ansioso Resistente \& EMT"s Autonomia

\begin{tabular}{|c|c|c|c|c|c|c|c|}
\hline \multirow{3}{*}{$\begin{array}{l}\text { VARIABLES DE } \\
\text { ESTUDIO }\end{array}$} & \multirow{3}{*}{ Pte. } & \multirow{3}{*}{$\mathrm{f}$} & \multicolumn{4}{|c|}{ Apego Ansioso Resistente } & \multirow[b]{3}{*}{$\mathrm{p}$} \\
\hline & & & \multicolumn{2}{|c|}{$\mathrm{Si}$} & \multicolumn{2}{|c|}{ No } & \\
\hline & & & $\mathrm{n}$ & $\%$ & $\mathrm{n}$ & $\%$ & \\
\hline \multirow{2}{*}{$\begin{array}{l}\text { Dependencia / } \\
\text { Incompetencia }\end{array}$} & $\mathrm{Si}$ & $\mathrm{n}$ & 0 & 0,0 & 12 & 21,4 & \multirow{2}{*}{0,057} \\
\hline & No & $\mathrm{n}$ & 14 & 100,0 & 44 & 78,6 & \\
\hline \multirow{2}{*}{$\begin{array}{l}\text { Vulnerabilidad al } \\
\text { Daño y Enfermedad }\end{array}$} & $\mathrm{Si}$ & $\mathrm{n}$ & 0 & 0,0 & 5 & 8,9 & \multirow{2}{*}{0,246} \\
\hline & No & $\mathrm{n}$ & 14 & 100,0 & 51 & 91,1 & \\
\hline \multirow{2}{*}{$\begin{array}{l}\text { Sumisión / Perdida } \\
\text { Individualidad }\end{array}$} & $\mathrm{Si}$ & $\mathrm{n}$ & 3 & 21,4 & 1 & 1,8 & \multirow{2}{*}{$0,005 *$} \\
\hline & No & $\mathrm{n}$ & 11 & 78,6 & 55 & 98,2 & \\
\hline \multirow{2}{*}{$\begin{array}{l}\text { Temor a Perder el } \\
\text { Control / Inhibición } \\
\text { Emocional }\end{array}$} & $\mathrm{Si}$ & $\mathrm{n}$ & 3 & 21,4 & 1 & 1,8 & \multirow{2}{*}{$0,005 *$} \\
\hline & No & $\mathrm{n}$ & 11 & 78,6 & 55 & 98,2 & \\
\hline
\end{tabular}

Pte. $=$ presencia de la variable $; \mathrm{f}=$ frecuencia absoluta; $\mathrm{p}$ calculada por el chi cuadrado

Fuente: Autores

Teniendo como base los datos arrojados, se observa un alto nivel de asociación entre la variable de Apego Ansioso Resistente y la variable EMT’s de Sumisión / Perdida de la Individualidad y Temor a Perder el Control / Inhibición Emocional (Tabla N 3).

Tabla 4. Tabla de contingencia Apego Ansioso Elusivo \& EMT’s Autonomía

\begin{tabular}{|c|c|c|c|c|c|c|c|}
\hline \multirow{3}{*}{$\begin{array}{l}\text { VARIABLES DE } \\
\text { ESTUDIO }\end{array}$} & \multirow{3}{*}{ Pte. } & \multicolumn{6}{|c|}{ Apego Ansioso Elusivo } \\
\hline & & $\mathrm{f}$ & \multicolumn{2}{|c|}{$\mathrm{Si}$} & \multicolumn{2}{|c|}{ No } & \multirow[b]{2}{*}{$\mathrm{p}$} \\
\hline & & & $\mathrm{n}$ & $\%$ & $\mathrm{n}$ & $\%$ & \\
\hline \multirow{2}{*}{$\begin{array}{l}\text { Dependencia / } \\
\text { Incompetencia }\end{array}$} & $\mathrm{Si}$ & $\mathrm{n}$ & 0 & 0,0 & 12 & 19,0 & \multirow{2}{*}{0,205} \\
\hline & No & $\mathrm{n}$ & 7 & 100,0 & 51 & 81,0 & \\
\hline \multirow{2}{*}{$\begin{array}{l}\text { Vulnerabilidad al } \\
\text { Daño y Enfermedad }\end{array}$} & $\mathrm{Si}$ & $\mathrm{n}$ & 0 & 0,0 & 5 & 7,9 & \multirow{2}{*}{0,439} \\
\hline & No & $\mathrm{n}$ & 7 & 100,0 & 58 & 92,1 & \\
\hline \multirow{2}{*}{$\begin{array}{l}\text { Sumisión / Perdida } \\
\text { Individualidad }\end{array}$} & $\mathrm{Si}$ & $\mathrm{n}$ & 1 & 14,3 & 3 & 4,8 & \multirow{2}{*}{0,303} \\
\hline & No & $\mathrm{n}$ & 6 & 85,7 & 60 & 95,2 & \\
\hline \multirow{2}{*}{$\begin{array}{l}\text { Temor a Perder el } \\
\text { Control / Inhibición } \\
\text { Emocional }\end{array}$} & $\mathrm{Si}$ & $\mathrm{n}$ & 1 & 14,3 & 3 & 4,8 & \multirow[b]{2}{*}{0,303} \\
\hline & No & $\mathrm{n}$ & 6 & 85,7 & 60 & 95,2 & \\
\hline
\end{tabular}

Pte. $=$ presencia de la variable; $\mathrm{f}=$ frecuencia absoluta; $\mathrm{p}$ calculada por el chi cuadrado Fuente: Autores

Teniendo como base los datos arrojados, no se observa un alto nivel de asociación entre la variable de Apego Ansioso Elusivo y la variable EMT’s (Tabla N 4).

Tabla 5. Tabla de contingencia Apego Extremo \& EMT’s Autonomía

Citación del artículo: Villa Londoño, A., Álvarez Posada, J. (2017). Relación entre el tipo de apego y los esquemas maladaptativos tempranos del área de la autonomía en una muestra de niños de 2 a 6 años de edad. Revista Katharsis, N 23, enero-julio 2017, pp. 18-33, Disponible en http://revistas.iue.edu.co/index.php/katharsis 


\begin{tabular}{|c|c|c|c|c|c|c|c|}
\hline \multirow{3}{*}{$\begin{array}{l}\text { VARIABLES DE } \\
\text { ESTUDIO }\end{array}$} & \multirow{3}{*}{ Pte. } & \multicolumn{6}{|c|}{ Apego Extremo } \\
\hline & & $\mathrm{f}$ & \multicolumn{2}{|c|}{$\mathrm{Si}$} & \multicolumn{2}{|c|}{ No } & \multirow[b]{2}{*}{$\mathrm{p}$} \\
\hline & & & $\mathrm{n}$ & $\%$ & $\mathrm{n}$ & $\%$ & \\
\hline \multirow{2}{*}{$\begin{array}{l}\text { Dependencia / } \\
\text { Incompetencia }\end{array}$} & $\mathrm{Si}$ & $\mathrm{n}$ & 6 & 40,0 & 6 & 10,9 & \multirow{2}{*}{$0,008 *$} \\
\hline & No & $\mathrm{n}$ & 9 & 60,0 & 49 & 89,1 & \\
\hline \multirow{2}{*}{$\begin{array}{l}\text { Vulnerabilidad al } \\
\text { Daño y Enfermedad }\end{array}$} & $\mathrm{Si}$ & $\mathrm{n}$ & 5 & 33,3 & 0 & 0,0 & \multirow{2}{*}{$0,000 *$} \\
\hline & No & $\mathrm{n}$ & 10 & 66,7 & 55 & 100,0 & \\
\hline \multirow{2}{*}{$\begin{array}{l}\text { Sumisión / Perdida } \\
\text { Individualidad }\end{array}$} & $\mathrm{Si}$ & $\mathrm{n}$ & 0 & 0,0 & 4 & 7,3 & \multirow{2}{*}{0,282} \\
\hline & No & $\mathrm{n}$ & 15 & 100,0 & 51 & 92,7 & \\
\hline \multirow{2}{*}{$\begin{array}{l}\text { Temor a Perder el } \\
\text { Control / Inhibición } \\
\text { Emocional }\end{array}$} & $\mathrm{Si}$ & $\mathrm{n}$ & 0 & 0,0 & 4 & 7,3 & \multirow[b]{2}{*}{0,282} \\
\hline & No & $\mathrm{n}$ & 15 & 100,0 & 51 & 92,7 & \\
\hline
\end{tabular}

Pte. $=$ presencia de la variable; $\mathrm{f}$ = frecuencia absoluta; $\mathrm{p}$ calculada por el chi cuadrado

Fuente: Autores

Teniendo como base los datos arrojados, se observa un alto nivel de asociación entre la variable de Apego Extremo y la variable EMT’s de Dependencia / Incompetencia y Sumisión / Perdida de la Individualidad (Tabla N 5).

\section{Discusión de resultados.}

El siguiente análisis se realiza a partir de la teoría de los Esquemas Maladaptativos Tempranos de Jeffrey Young tomando como base la información obtenida a partir de los resultados de las pruebas y su posterior procesamiento a través del software SPSS 22.

De acuerdo con los resultados de la investigación, se observa relación entre el tipo de apego y algunos EMT del área de la autonomía. Se rechaza entonces la Hipótesis nula $\left(\mathrm{H}_{0}\right)$, y se confirma la Hipótesis Alternativa $\left(\mathrm{H}_{1}\right)$, la que indica que si existen EMT's de dicha área relacionados con el tipo de apego del niño.

Para Jeffrey Young, las influencias destructivas de la infancia interaccionan con el temperamento y conforman las trampas vitales. El temperamento puede determinar solo de manera parcial la manera en que el niño es tratado por los padres. A pesar de tener un mismo entorno, los niños pueden reaccionar de forma muy diferente: ambos pueden ser maltratado, pero quizá uno reaccionara de manera pasiva, mientras que el otro luchará en contra (Young, 2012). Dichas influencias destructivas pueden entenderse como los estilos parentales que los

Citación del artículo: Villa Londoño, A., Álvarez Posada, J. (2017). Relación entre el tipo de apego y los esquemas maladaptativos tempranos del área de la autonomía en una muestra de niños de 2 a 6 años de edad. Revista Katharsis, N 23, enero-julio 2017, pp. 18-33, Disponible en http://revistas.iue.edu.co/index.php/katharsis 
cuidadores del niño ejercen sobre el mismo, ya sea a partir de pautas ambivalentes, elusivas o excesivamente controladoras.

A continuación, se exponen aspectos relacionados con los vínculos de apego y su correlación con EMT del área de la autonomía:

Específicamente con respecto a la relación entre el tipo de apego seguro y los Esquemas Maladaptativos Tempranos del área de la autonomía, se observa un alto nivel de asociación entre la variable de Apego Seguro y la variable EMT's de Vulnerabilidad al Daño y a la Enfermedad, Sumisión / Perdida de la Individualidad y Temor a Perder el Control / Inhibición Emocional.

El EMT de vulnerabilidad al daño y a la enfermedad hace referencia al exagerado temor a que la calamidad o el desastre esté a punto de golpearnos en cualquier momento, a nivel natural, criminal, salud, y que uno es incapaz de protegerse a sí mismo (Young, 1993).

Llama la atención que este esquema haya correlacionado con el apego seguro, dato que parece contradecir la vivencia de seguridad típica del infante con respecto a la disponibilidad y sensibilidad de sus cuidadores, pero, por otro lado, esta relación podría explicarse en la medida en que se comprende que la población investigada va de los 2 a los 6 años de edad, y que en estas fases el proceso de autoafirmación y seguridad del sujeto en sí mismo está en proceso de construcción. También habría que tener en cuenta las características del ambiente social en donde estos niños se están desarrollando y madurando. Igualmente, aunque gran cantidad de niños en esta población presenten sentimientos de inseguridad frente a su integridad física, también es cierto que presentan confianza en que si estuviesen en peligro contarían con el apoyo de sus cuidadores para ponerse a salvo. Dicho esquema de vulnerabilidad entraría a formar parte del llamado por Bowlby modelo representacional (Bowlby, 1980), como un aspecto de auto referenciación del niño de sí mismo y de sus relaciones con los demás.

El EMT de Sumisión / Perdida de la Individualidad hace referencia a la entrega excesiva del control sobre nuestras propias decisiones, preferencias y expresión emocional en las manos de los demás; generalmente para evitar la ira, las represalias o el abandono. Conlleva la percepción de que nuestros propios sentimientos y deseos no son válidos o

Citación del artículo: Villa Londoño, A., Álvarez Posada, J. (2017). Relación entre el tipo de apego y los esquemas maladaptativos tempranos del área de la autonomía en una muestra de niños de 2 a 6 años de edad. Revista Katharsis, N 23, enero-julio 2017, pp. 18-33, Disponible en http://revistas.iue.edu.co/index.php/katharsis 
importantes para los demás, lo cual implica su sacrificio para satisfacer los de los otros. Con frecuencia conduce a dificultades para establecer metas y dirección en la vida, así como a fallas en el reconocimiento de mis propias necesidades, y termina generando la ira hacia el subyugador (Young, 2012).

$\mathrm{Si}$ bien algunos niños objeto de este este estudio mostraron un apego seguro con respecto a sus cuidadores, también se observa en parte de este bloque de población la presencia del esquema de sumisión / perdida de la individualidad. Esto podría explicarse como un intento por parte de algunos niños por mantener la disponibilidad de los cuidadores a expensas de sus propias necesidades, patrón que de continuar durante el desarrollo y de ser reforzado por los padres traería graves consecuencias en los niños como la idea de que el cuidado y amor que se recibe está condicionado a la sumisión y subyugación y que por lo tanto es un amor condicional.

El EMT de Temor a Perder el Control / Inhibición Emocional hace referencia al temor a que uno, de manera inminente e involuntaria, perderá el control de su propia conducta, sus impulsos, sus emociones, su cuerpo o su mente. Esto generalmente si se permite sentir y expresar sus emociones (Young, 1993).

Acá se observa la presencia de otro EMT que estaría en contraposición de un apego seguro. Los niños objeto de estudio si bien muestran un sentimiento de confianza con respecto a la sensibilidad, disponibilidad y colaboración por parte de sus cuidadores, también muestran dificultades según este esquema para expresar sus emociones de manera tranquila y fluida y/o para confiar en su capacidad para expresar sus emociones y usarlas de manera saludable y adaptativa.

Con respecto a la relación entre el tipo de apego ansioso resistente los Esquemas Maladaptativos Tempranos del área de la autonomía, se observa un alto nivel de asociación entre la variable de Apego Ansioso resistente y la variable EMT's de Sumisión / Perdida de la Individualidad y Temor a Perder el Control / Inhibición Emocional.

Sumisión / Perdida de la Individualidad: Teniendo en cuenta que en el apego Ansioso Resistente el individuo adopta falta de fiabilidad y que a la vez esto genera gran ansiedad hay que tener en cuenta que estos niños no saben qué hacer para expresar sus necesidades y ser

Citación del artículo: Villa Londoño, A., Álvarez Posada, J. (2017). Relación entre el tipo de apego y los esquemas maladaptativos tempranos del área de la autonomía en una muestra de niños de 2 a 6 años de edad. Revista Katharsis, N 23, enero-julio 2017, pp. 18-33, Disponible en http://revistas.iue.edu.co/index.php/katharsis 
atendidos, entonces optan por llorar más fuerte y durante más tiempo, gritan o se aferran de manera ansiosa a la figura de apego en actitud desesperada, sus actitudes son de sumisión y de pérdida total de individualidad pues la angustia prima sobre cualquier otra vivencia emocional.

El esquema de Temor a Perder el Control / Inhibición Emocional se asocia directamente al Apego ansioso resistente, esto puede explicarse ya que el niño no encuentra la respuesta que espera y necesita por parte de su cuidador, pues cualquier comportamiento seguramente molestaría a su madre, que le rechazaba o castigaba sin entender que tras él se esconde el dolor de no sentirse suficiente amado ni valioso para ella. Esto conlleva a que el infante presenta la vivencia emocional de que en el futuro nadie podrá quererlo realmente lo que tiende a llevarlo a una sensación de pérdida de control sobre el ambiente y se verá obligado a inhibir sus respuestas emocionales para evitar molestar a los demás y, así, aumentar las probabilidades de permanencias de los otros a su lado.

Específicamente con respecto a la relación entre el tipo de Apego Elusivo y los Esquemas Maladaptativos Tempranos del área de la autonomía, no se observa un alto nivel de asociación entre dichas variables.

Teniendo como base los datos arrojados, se observa un alto nivel de asociación entre la variable de Apego Extremo y la variable EMT’s de Dependencia / Incompetencia y Sumisión / Perdida de la Individualidad.

Dependencia / Incompetencia se refiere a la creencia de que uno es incapaz de manejar sus responsabilidades cotidianas de forma competente, sin ayuda considerable de los demás. La creencia de que uno es incapaz de funcionar solo, por su cuenta (Young, 1993).

El apego extremo formado con la inadecuada libertad para lograr una fluida independencia puede generar en su dinámica la creencia en el niño de que es incapaz de manejar sus responsabilidades cotidianas de forma competente y que es inepto para actuar por su propia cuenta.

Con respecto al esquema de Sumisión / Perdida de la Individualidad se puede explicar su presencia en los niños con apego extremo, por la negociación a la que los mismos se ven obligados para satisfacer las demandas de los cuidadores y así obtener por parte de ellos la

Citación del artículo: Villa Londoño, A., Álvarez Posada, J. (2017). Relación entre el tipo de apego y los esquemas maladaptativos tempranos del área de la autonomía en una muestra de niños de 2 a 6 años de edad. Revista Katharsis, N 23, enero-julio 2017, pp. 18-33, Disponible en http://revistas.iue.edu.co/index.php/katharsis 
validación para realizar alguna actividad relacionada con su deseo de autonomía. Por otro lado, el niño con este esquema y este tipo de apego sentirá que para poder mantener los cuidados a los que ya se ha hecho dependiente por parte de su cuidador, deberá evitar a toda costa hacer demandas a partir de necesidades que sean amenazantes para su figura de apego, so pena de abandono, culpa o en algunos casos maltrato. Es importante mencionar que el comportamiento de las madres que facilitan un apego extremo puede ser el fruto de una estrategia no necesariamente consiente dirigida a aumentar la dependencia del niño, asegurando su cercanía y utilizándolo a su vez como figura de apego (Cassidy y Berlín, 1994), lo que interfiere con la propia necesidad del niño de explorar el mundo que lo rodea más allá de su madre y forzándolo a desarrollar como estrategia la conducta dependiente para lograr la atención.

\section{Conclusiones.}

El tipo de apego está directamente relacionado con patrones que comprometen a recuerdos, sensaciones corporales, emociones y cogniciones que el sujeto posee acerca de sí mismo a través de las relaciones que establece con otros durante su infancia, mientras esto sucede, los EMT's también van tomando forma convirtiéndose en estructuras estables y duraderas. Es de esperarse entonces que la H1 se confirme pues mientras los EMT's se establecen y desarrollan el estilo parental y el tipo de apego que de allí se desprende van dando aportes fundamentales a su estructuración.

Es importante para futuras investigaciones tener en cuenta las características del ambiente y los peligros a los cuales los niños pudieran estar expuestos para lograr comprensiones más precisas y acordes a los resultados.

Los cuidadores deben ser supremamente cautelosos en su interacción con los niños y demostrarles de manera autentica que el amor y cuidados que les profesan son incondicionales y que no tienen que ver con una postura sumisa y subyugada para mantener a los padres contentos, satisfechos y dispuestos. Igualmente, los padres deben esforzarse por ayudarle a sus hijos a desarrollar un sentimiento de confianza en sus propias emociones y de que estas son herramientas que pueden ser de suprema utilidad en la resolución de problemas y en las

Citación del artículo: Villa Londoño, A., Álvarez Posada, J. (2017). Relación entre el tipo de apego y los esquemas maladaptativos tempranos del área de la autonomía en una muestra de niños de 2 a 6 años de edad. Revista Katharsis, N 23, enero-julio 2017, pp. 18-33, Disponible en http://revistas.iue.edu.co/index.php/katharsis 
relaciones interpersonales. La noción condicional del amor a expensas como se dijo anteriormente de las propias necesidades o de la capacidad para expresar emociones no es en absoluto una idea saludable y los cuidados deben estar pendientes de cómo se está dando el desarrollo de la inteligencia emocional del niño. Es muy importante que los padres faciliten y favorezcan la expresión emocional de los niños.

Para los niños es fundamental que los cuidadores faciliten su autonomía para que tengan la oportunidad de desarrollar un profundo sentimiento de autoconfianza y autoeficacia. Estos cuidados no pueden ser condicionales y los padres deben esforzarse en desarrollar un sentimiento de seguridad en el infante y que este tenga la certeza de que puede expresar sus emociones y necesidades sin que haya una amenaza tacita o explicita que hacerlo conllevará al abandono, la culpa o el maltrato.

El modelo focalizado en esquemas argumenta que los esquemas y los estilos de afrontamiento desadaptativas están en el centro de un extenso rango de trastornos crónicos (Caro, 1997). Lo anterior indica que la presencia de estos EMT en los niños estaría directamente relacionada con una mayor vulnerabilidad por parte de éstos a desarrollar trastornos a lo largo de la infancia y/o la vida adulta, y teniendo en cuenta los EMT que correlacionaron podría hablarse de problemáticas relacionadas con la personalidad dependiente, alexitimia por el bloqueo emocional, trastornos somatomorfos por la incapacidad para procesar las emociones, problemas de autoeficacia y el posible desarrollo de pautas de sumisión que podrían a su vez generar patrones pasivo agresivos des adaptativos.

Por otro lado es importante tener en cuenta que aquellos individuos que tienen una mejor historia de apego es más probable que tengan relaciones amorosas más satisfactorias (Feeney y Noller, 1990), y que por el contrario los niños que han sido criados bajo pautas de apego ambivalentes, elusivas o extremas también serán más vulnerables a desarrollar problemáticas relacionadas con las relaciones de pareja, en donde los EMT acá mencionados jugarían un papel importante en el desarrollo de pautas poco viables dentro de su esquema interpersonal.

Otro dato importante a tener en cuenta es que las pautas recibidas por los niños tenderán a transmitirse intergeneracionalmente de estos a sus propios hijos, pues existen

Citación del artículo: Villa Londoño, A., Álvarez Posada, J. (2017). Relación entre el tipo de apego y los esquemas maladaptativos tempranos del área de la autonomía en una muestra de niños de 2 a 6 años de edad. Revista Katharsis, N 23, enero-julio 2017, pp. 18-33, Disponible en http://revistas.iue.edu.co/index.php/katharsis 
investigaciones que logran demostrar que la capacidad predictiva que las representaciones maternas tienen sobre el tipo de apego que establecen sus hijos ronda el 80\% ((Benoit y Parker, 1994; Fonagy, Steele y Steele, 1991), facilitando así en caso de los tipos de apego no seguros el mantenimiento de los EMT's a través de varias generaciones, ya sea manifestándose estos de manera directo o a través de conductas por sobrecompensación (Young, 2012). Esto es importante tenerlo en cuenta con el fin de realizar intervenciones de tipo preventivo en poblaciones similares, ya que dicha transmisión intergeneracional es real mas no inevitable.

\section{Referencias}

Ainsworth, M., Bell, S. (1970). Apego, exploración y separación, ilustrados a través de la conducta de niños de un año en una situación extraña. En J. Delval (Comp.). Lecturas de psicología del niño. Vol. 1. Madrid: Alianza

Benoit, D., Parker, K. (1994). Stability and transmission of attachment across three generations. Child Development, 65, 1444-1456. Disponible en https://www.ncbi.nlm.nih.gov/pubmed/7982361

Bowlby, J. (1980). La pérdida afectiva. Tristeza y depresión. Buenos Aires: Paidós, 1984

Bowlby, J. (1980). Una base segura. Buenos Aires: Paidós, 1980

Cassidy, J., Berlin, L. (1994). The insecure/ambivalent pattern of attachment: Theory and research. Child Development, 65, 971-994. Disponible en https://www.ncbi.nlm.nih.gov/pubmed/7956474

Caro, I. (1997). Manual de psicoterapias cognitivas. Madrid: Paidos. Pag. 223, 232

Feeney, J., Noller, P. (1990). Attachment style as a predictor of adult romantic relationships. Journal of Personality and Social Psychology, 58, 281-291. Disponible en http://psycnet.apa.org/index.cfm?fa=buy.optionToBuy\&id=1990-14609-001

Fonagy, P., Steele, H., Steele, M. (1991). Maternal representations of attachment during pregnancy predict the organization of infant-mother attachment at one year of age.

Citación del artículo: Villa Londoño, A., Álvarez Posada, J. (2017). Relación entre el tipo de apego y los esquemas maladaptativos tempranos del área de la autonomía en una muestra de niños de 2 a 6 años de edad. Revista Katharsis, N 23, enero-julio 2017, pp. 18-33, Disponible en http://revistas.iue.edu.co/index.php/katharsis 
Child Development, 62, 891-905, Disponible en https://www.ncbi.nlm.nih.gov/pubmed/1756665

Young, J., Kloslo, J. (2012). Reinventa tu vida. Barcelona: Paidós.

Young, J. (1999). Terapia centrada en esquemas para trastornos de personalidad. Barcelona: Paidós.

Citación del artículo: Villa Londoño, A., Álvarez Posada, J. (2017). Relación entre el tipo de apego y los esquemas maladaptativos tempranos del área de la autonomía en una muestra de niños de 2 a 6 años de edad. Revista Katharsis, N 23, enero-julio 2017, pp. 18-33, Disponible en http://revistas.iue.edu.co/index.php/katharsis 